\title{
ИЗМЕРЕНИЕ ДИНАМИЧЕСКИХ ПАРАМЕТРОВ СЕРДЕЧНО-СОСУДИСТОЙ СИСТЕМЫ ЧЕЛОВЕКА
}

\author{
В. В. Чернецкий
}

Институт кибернетики им. В. М. Глушкова НАН Украины

Розглянуто фонендоскопічний метод вимірювання тиску потоку крові на стінки кровоносних судин і визначення швидкості цього потоку.

Сердечно-сосудистая система (ССС) предназначена для выполнения транспортной функции доставки крови к каждой клетке организма и обеспечивает пространственно-временную реализацию главных физических и физиологических процессов в организме человека. В движение поток крови приводится силами, которые генерируются сердцем. Поддержание непрерывности потока крови - основная функция ССС. Сила $F(t)$ непосредственно создается мускулатурой сердца и образует поток крови, который описывается транспортной функцией $V(t)$. Изложенное представляет собой теорию работы ССС человека.

Система кровоснабжения человека представляет собой сложный механизм, состояние которого оценивается в настоящее время электрокардиограммой или фонограммой. Однако электрокардиограмма отражает только электрическую активность сердечной мышцы, а функциональная способность сердца в качестве насоса определяется по ЭКГ косвенным способом. Достоверное представление движения крови в ССС может дать только прямое приборное измерение физических параметров потока крови в сосудах с последующим его отображением. Оптимальным может быть неинвазивное измерение мгновенных значений давления частиц крови на стенки сосудов в выбранной точке, расположенной близко к поверхности тела. В CCC сила $F$ действует в форме давления $P_{k}$, которое равномерно распределено по площади $S$ кровеносного сосуда $F=P_{k} S$. Частицы крови в этом сечении приводятся в движение со скоростью $V_{\kappa}$. Параметры $P_{\kappa}$ и $V_{\kappa}$ являются основными динамическими параметрами ССС.

В Институте кибернетики разработано устройство для измерения и отображения динамических параметров ССС $P_{\kappa}$ и $V_{k}$, которое реализует фонендоскопический метод измерения мгновенных значений давления крови на стенки сосудов. Задача определения параметров движения крови в сосудах решена посредством измерения процесса $F(t)=P_{k}(t) S$. До недавнего времени непосредственное измерение давления $P_{\kappa}(t)$ было невозможно по причине отсутствия средств измерения необходимой чувствительности и быстродействия. Аппаратное измерение $P_{k}$ (t) выполняется устройством на основе цифрового микрофона давления, имеющего цифровую чувствительность существенно более высокую, чем стандартный порог слышимости $P_{o}=2 \cdot 10^{-5} \mathrm{H}^{\prime} \mathrm{M}^{2}$. В свою очередь проблема создания цифрового микрофона давления потребовала разработки способа измерения интенсивности выходных сигналов измерительного преобразователя давления, амплитуда которых существенно ниже уровня теплового шума на входе микрофонного усилителя. Успех этой работы обеспечен изобретением электромагнитного усилителя подшумовых сигналов [1], который имеет чувствительность к входному сигналу на несколько порядков выше, чем у обычных полупроводниковых усилителей, а также разработкой полевых функциональных преобразователей аналоговых гармонических сигналов в цифровую форму через преобразование их амплитуды в интервал времени. Работа усилителей и преобразователей основана на едином принципе управления магнитным полем формой электрического тока в проводниках [2]. В разработке использован наиболее эффективный путь достижения максимальной чувствительности, который состоит в исполнении цифрового измерительного преобразования, основанного на временном развертывающем преобразовании магнитного сопротивления и квантовании интервала времени.

Для преобразования измеряемых величин в интервал времени, который задается разностью фаз низкочастотных гармонических сигналов, используется процесс накопления энергии магнитного поля в магнетике и процесс ее преобразования в тепло в проводнике, индуктивно связанном с магнетиком. Высокая чувствительность способа измерения перемещения мембраны цифрового микрофона с магнитной индукцией достигается благодаря тому, что модуляция магнитного сопротивления магнитопровода перемещением мембраны микрофона и преоб-

(C) В. В. Чернецкий 
разование ее в фазовый сдвиг тока относительно фазы э.д.с. направлены на реализацию резонансного преобразования энергии электрического поля внешнего источника гармонической э.д.с. в энергию магнитного поля проводника, а последней в тепло. Если выполняется условие равенства процессов резонансного преобразования энергии, то в проводнике с током отсутствует фазовый сдвиг между током и э.д.с. В таком режиме происходит полное преобразование электромагнитной энергии в теплоту и достигается максимальная цифровая чувствительность измерения перемещения мембраны микрофона, которая обусловлена тем, что небольшая модуляция магнитного потока перемещением мембраны вызывает максимальную фазовую модуляцию тока в проводнике.

Для определения давления потока крови на стенки кровеносных сосудов в устройстве для измерения динамических параметров ССС используется фонендоскопический метод измерения. Устройство содержит точечный фонендоскоп, цифровой микрофон, интерфейсный блок и микропроцессор. Цифровой микрофон содержит мембрану из магнитомягкого материала, расположенную между двумя чашкообразными ферритовыми магнитопроводами. Акустический канал фонендоскопа нагружен на мембрану микрофона. В одной и другой чашках микрофона расположены катушки индуктивности, которые вместе с двумя первичными обмотками трансформатора образуют мост, питающийся гармоническими сигналами возбуждения магнитного поля в промежутке между ферритом и мембраной.

При измерении фонендоскоп располагается над кровеносным сосудом, в котором необходимо определить давление потока крови на его стенки. Поток крови деформирует стенку сосуда, которая вместе с прилегающими к ней тканями служит мембраной фонендоскопа. Акустический сигнал фонендоскопа передается на вход цифрового микрофона, который выдает в интерфейсный блок информационные сигналы о величине давления с частотой возбуждения микрофона. Акустическое давление на мембрану микрофона пропорционально давлению потока крови на стенки сосуда. В интерфейсном блоке на базе полевого сигнального процессора осуществляется преобразование информационных гармонических сигналов в коэффициенты Фурье в течение одного периода гармонического сигнала возбуждения цифрового микрофона. Дальнейшая математическая обработка результатов измерения возложена на микропроцессор и его программно ориентированное обес- печение. Цифровые отсчеты давления потока крови на стенки сосудов отображаются в виде циклограммы давления $P_{\kappa}(t)$ (рис. 1,a). Также в реальном времени выполняется цифровое интегрирование циклограммы давления, и результат вычисления отображается в виде циклограммы скорости потока крови $V_{\kappa}(t)($ рис. $1, б)$. Данные измерения и вычисления допускают параметризацию интенсивности процессов $P_{\kappa}(t)$ и $V_{k}(t)$ по их продолжительности. На циклограмме давления отмечаются максимальное давление $P_{m}$, соответствующее максимальному давлению в сосуде и, соответственно, в желудочке сердца, а также максимальное давление торможения потока крови $P_{T}$, соответствующее максимальному давлению клапана желудочка. Система параметров, служащих для диагностики работы ССС, имеет такую структуру. Ее физической величиной, образующей систему измеряемых и вычисляемых параметров, являются давление или сила, приведенная к единице площади, скорость потока крови, а также порядок и продолжительность фаз силового взаимодействия потока крови с мышцами сердца и стенками кровеносных сосудов.

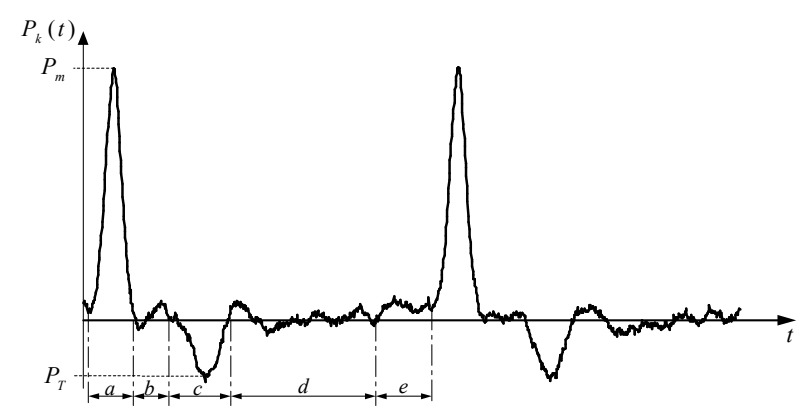

a

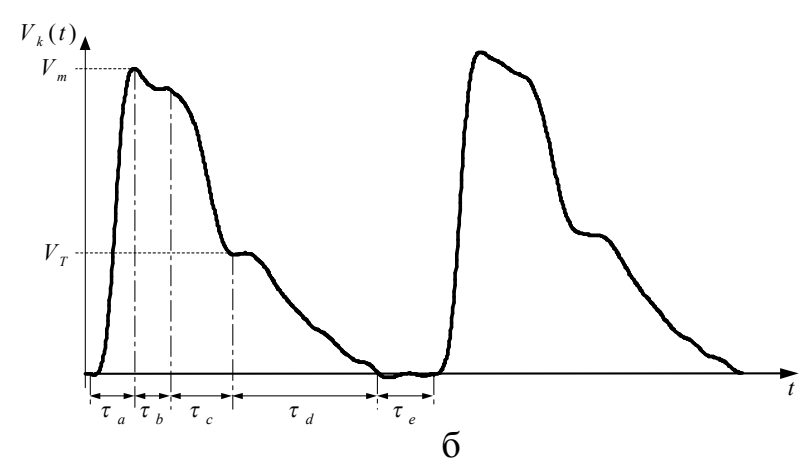

Puc. 1. Цифровые циклограммы давления и скорости потока крови.

Продолжительность фаз связана с важнейшими физиологическими процессами и дает достоверную диагностику работы сердечно-сосудистой системы по величине отклонения фазовых интервалов време- 
ни от нормы. Благодаря цифровому измерению фазовых интервалов исключается влияние индивидуальных отклонений интенсивности влияния механических процессов на качество их диагностики. Для точного измерения силовых параметров имеет определяющее значение высокая цифровая чувствительность измерения силы, а для точности отображения динамики процесса - частота его дискретизации. Высокая чувствительность и временное разрешение дают возможность получить точные

\section{Литература}

1. Патент № 87027. Україна. Електромагнітний підсилювач напруги / О. Д. Бех, В. В. Чернецький, В. В. Слшанський. Опубл.10.06.2009.--Бюл. № 11.

2. Бех А. Д. Автоматический контроль физических пара- значения измеряемых и вычисляемых параметров, которые исчерпывающим образом позволяют судить о физических процессах, происходящих в сердечнососудистой системе. Циклограмма скорости потока крови в сосуде соответствует графику пульсовой волны.

Визуализация силового действия сердца на поток крови в сосудах и скорости потока является объективной основой для диагностики заболеваний и наблюдения действия лекарств.

метров монет / А. Д. Бех, В. В.Чернецкий, В. В. Елшанский // Комп'ютерні засоби, мережі та системи. -2004. - № 3.C. 24-34. 